\title{
Radiotherapy effects on brain/bone metastatic adenocarcinoma lung cancer and the importance of EGFR mutation test
}

\author{
B. L. QU $U^{\sharp, *}$, B. N. CAI", W. YU, F. LIU, Y. R. HUANG, Z. J. JU, X. S. WANG, G. M. OU, L. C. FENG \\ Department of Radiotherapy, PLA General Hospital, Beijing 100853,China \\ ${ }^{*}$ Correspondence: qubaolin_bj@sina.cn \\ "Contributed equally to this work.
}

Received May 26, 2015 / Accepted August 24, 2015

\begin{abstract}
This study proposed to retrospectively analyze the efficacy of radiotherapy on brain/bone metastases in patients with stage IV lung adenocarcinoma and to evaluate the correlation between overall survival after radiotherapy and other factors including metastatic sites and EGFR mutation status. 115 patients with Stage IV lung adenocarcinoma admitted to our center from March, 2011 to December, 2013 were enrolled. They presented with metastases to no other solid organs except the bone or brain and had received no prior treatment. 50 patients received EGFR mutation test with 32 detected as EGFR mutant and 18 wild-type. Patients with brain metastases were treated with 40 Gy whole brain irradiation (WBI) in 2 Gy fractions; patients with bone metastases were treated with $30 \mathrm{~Gy}$ local irradiation in $3 \mathrm{~Gy}$ fractions or $40 \mathrm{~Gy}$ in 2 Gy fractions. All the patients received systemic therapy during or after radiotherapy and 68 received targeted therapy.The median overall survival of patients with solitary brain metastases, solitary bone metastases or combined brain and bone metastases were 8.50 months, 8.50 months and 9.50 months respectively, revealing no significant difference $(\mathrm{p}=0.57)$. The median overall survival of patients with EGFR mutations was 10.25 months, longer than the 8.75 months of patients without EGFR mutations, revealing no significant difference $(\mathrm{p}=0.57)$. The median overall survival of EGFR mutant patients with solitary bone metastases, solitary brain metastases or combined brain and bone metastases were 7.50 months, 10.50 months and 11.50 months respectively, revealing no significant difference ( $\mathrm{p}=0.91) .36$ patients with untested EGFR mutation status received EGFR-TKI. Among EGFR mutant patients, 10 didn't receive targeted therapy; 8 were administered Erlotinib and 14 Gefitinib with median overall survival of 10.25 months and 14.5 months, showing no significant difference $(p=0.11)$ between the two drugs. When patients with stage IV lung adenocarcinoma have been treated by early radiotherapy, the overall survival doesn't correlate with metastatic sites. Radiotherapy could extend survival for EGFR mutant patients with stage IV lung adenocarcinoma. EGFR mutation test should be performed before treatment of the disease.
\end{abstract}

Key words: radiotherapy, metastases, lung adenocarcinoma, EGFR mutations

Metastatic sites and survival in lung cancer are influenced by sex, histological subtype, and age at diagnosis. The most frequent metastatic sites are the bone and brain [1]. Nearly $10 \%$ of non-small cell lung cancer (NSCLC) patients present with brain metastases as first diagnosis and another 30\%-50\% develop brain metastases during disease progression [2]. Blood brain barrier is a physiological obstruction to the delivery of chemotherapy, therefore radiotherapy remains the first choice in treatment of brain metastases [2,3]. Patients who present with Exon 19 deletions and Exon 21 point mutations (L858R mutation) in epidermal growth factor receptor have better prognosis than those without EGFR mutations $[4,5,6]$. Existing studies haven't come to a consistent conclusion that in lung adenocarcinoma cases with brain metastases, EGFR mutant patients have better prognosis than patients without EGFR mutations $[7,8]$, while it has been agreed that radiation of brain metastases could enhance local control of metastatic brain lesions [9]. In recent years epidermal growth factor receptor tyrosine kinase inhibitors (EGFR-TKI) including Gefitinib and Erlotinib have been widely used in treatment of lung adenocarcinoma with EGFR mutations, since there are studies suggesting these inhibitors can be slightly transported across $\mathrm{BBB}$, and make treatment of brain metastases by drugs possible $[10,11]$. One of the most frequent metastatic sites is the bone [1]. Painful bone metastases could lead to local bone destruction and reduce life quality of patients. 


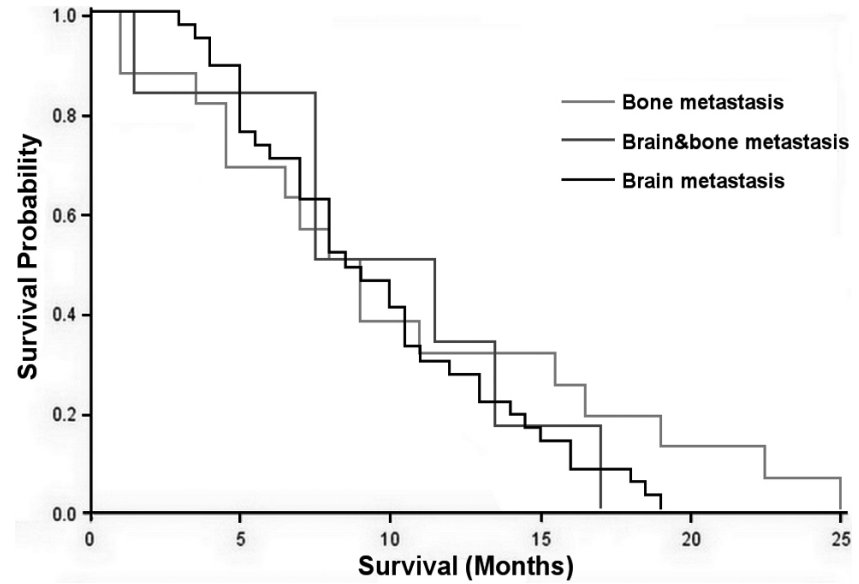

Figure 1. Brainbone metastases and median overall survival.

While radiotherapy remains an effective method for treating bone metastases, patients could also get survival benefit from EGFR-TKI [14]. This study gave a retrospective analysis on stage IV lung adenocarcinoma patients with brain/bone metastases who were treated in our center, and evaluated the correlation between overall survival (OS) after radiotherapy and other factors including EGFR mutations, and metastatic.

\section{Patients and methods}

115 patients with Stage IV lung adenocarcinoma admitted from March, 2011 to December, 2013 were enrolled. They presented with metastases to no other solid organs except the bone or brain and had received no prior surgery, radiotherapy, chemotherapy or TKI treatment. 60 patients had brain metastases, 42 bone metastases, 13 combined brain and bone metastases; 69 male and 46 female. The median age of the participants was 56.8 years (35-82). 50 patients received EGFR mutation test by DNA sequencing, which revealed that 32 patients had Exon 19 deletions and L858R mutation while 18 had no EGFR mutations(Table1). Patients with brain metastases were treated with 40 Gy whole brain irradiation (WBI) in 2 Gy fractions; patients with solitary bone metastases or combined brain and bone metastases were treated with $30 \mathrm{~Gy}$ local irradiation in $3 \mathrm{~Gy}$ fractions or $40 \mathrm{~Gy}$ in $2 \mathrm{~Gy}$ fractions. During or after radiotherapy all the patients received systemic therapy and 68 of them received targeted drug therapy including Gefitinib and Erlotinib.

The follow-up lasted until September 12, 2014. Loss to follow-up was calculated by the patient's last visit. KaplanMeier estimator (also known as product limited estimator) and Log-rank test were performed to evaluate the association between OS and other factors including brain/bone metastases, EGFR mutations, brain/bone metastases in EGFR mutant patients. SPSS 19.0 was adopted.

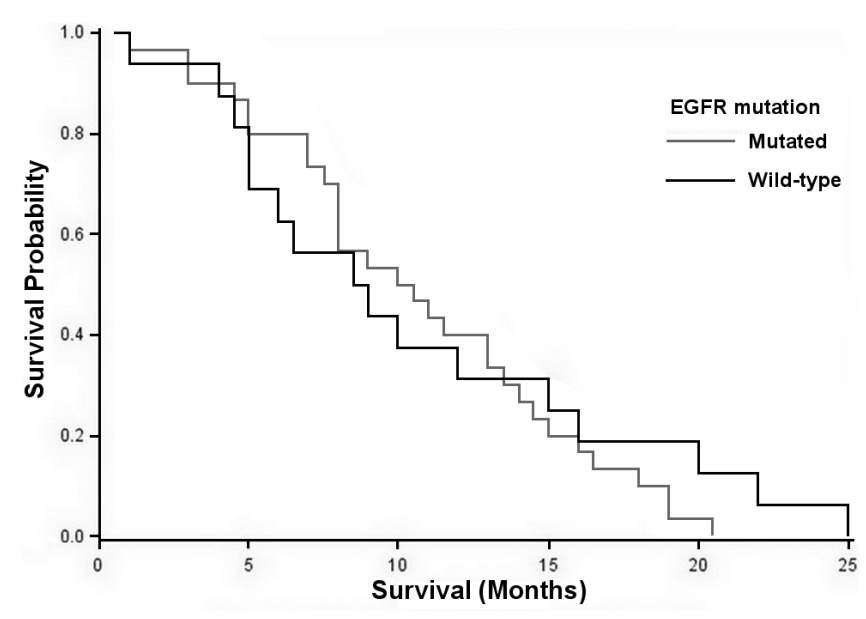

Figure 2. EGFR mutation status and median overall survival.

\section{Results}

Brain/bone metastases and median overall survival. The median overall survival of patients with solitary brain metastases, solitary bone metastases or combined brain and bone metastases were 8.50 months, 8.50 months and 9.50 months, respectively. As indicated in Figure 1, Log-rank test showed the three groups had no significant difference in survival rate $(\mathrm{p}=0.57)$. (Figure 1)

EGFR mutation status and median overall survival. The median overall survival of EGFR mutant patients was 10.25 months, longer than the 8.75 months of patients without mutations. As indicated in Figure 2, Log-rank test showed no significant difference between the two groups ( $\mathrm{p}=0.57$ ). (Figure 2)

Table 1. Patient and Tumor Characteristics $(n=115)$

\begin{tabular}{lc}
\hline Age (yrs) (mean+SD) range & $56.8 \pm 10.1(35-82)$ \\
Sex & \\
Male & $69(60.0 \%)$ \\
Female & $46(40.0 \%)$ \\
Metastases & \\
Brain & $60(52.2 \%)$ \\
Bone & $42(36.5 \%)$ \\
Brain+bone & $13(11.3 \%)$ \\
EGFR mutation testing & \\
Mutation & 32 \\
Wild-type & 18 \\
Unknown & 65 \\
Differentiation & \\
Low & 26 \\
Moderatel & 40 \\
High & 2 \\
Unknown & 47 \\
\hline
\end{tabular}




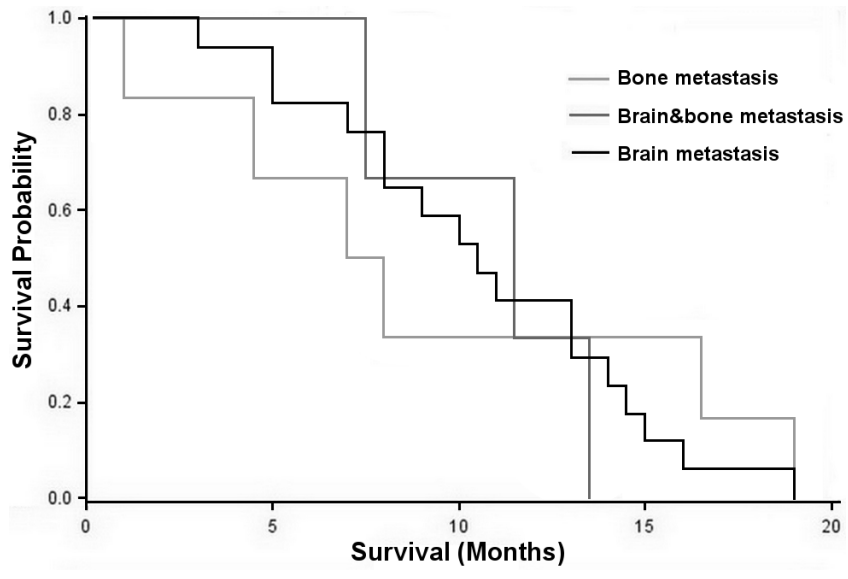

Figure 3. Median survival and brainbone metastases in EGFR mutant patients.

Median survival and brain/bone metastases in EGFR mutant patients. The median overall survival of EGFR mutant patients with solitary bone metastases, solitary brain metastases or combined brain and bone metastases were 7.50 months, 10.50 months and 11.50 months, respectively. Logrank test detected no significant difference in survival rate $(\mathrm{p}=0.91)$ among the three groups. (Figure 3$)$

Targeted drug therapy and median survival. 8/32 EGFR mutant patients received Erlotinib and 14 received Gefitinib. The median overall survival of the two subgroups were 10.25 months and 14.5 months respectively, showing no significant difference $(p=0.11)$.

\section{Discussion}

Lung adenocarcinoma is one of the most common malignant cancers in respiratory system. 38\% of the patients will have hematogenous metastases and 19\% have metastases to multiple sites. The most frequent metastatic sites include the nervous system, bone, liver, respiratory system and adrenal gland. Brain metastases had better prognosis than bone metastases and liver metastases but the patients' median survival was just 3-5 months $[1,2]$. It has been testified that EGFR mutations are often found in women patients, patients at the age from 51 to 60 , non-smoker patients and patients with adenocarcinoma or bronchioloalveolar carcinoma. The median survival time of patients with Exon 19 deletions and L858R mutation is 3.1 years, longer than the 1.6 years of wild-type patients ( $\mathrm{p}=0.001$ ), while no significant difference was found between Exon 19 deletions and L858R mutation [4,5]. Mak et al. [6] carried out a multivariate analysis on 1445 patients with lund adenocarcinoma, of whom 418 (29\%) had EGFR mutations and 1027 were wild-type. The local recurrence (LRR) rate was significantly lower in EGFR mutant group than in wild-type group ( $24 \%$ vs $46 \%$ ) with median survival time of 61.2 months and 34.7 months respectively.
While stage IV lung adenocarcinoma patients got survival benefits from radiotherapy, their survival time could also be affected by how the primary tumor had been controlled [2]. As EGFR mutant patients could benefit from EGFR-TKI therapy, EGFR mutation test by DNA sequencing may be included as part of the clinical care for stage IV lung adenocarcinoma [4]. Research carried out by Fujimoto et al. [15] indicated that EGFR mutant patients with stage IV lung adenocarcinoma treated by EGFR-TKI had better response than EGFR wild-type patients who received chemotherapy as first-line treatment, with progression-free survival (PFS) of 8.5 months and 3.6 months respectively. They also found better prognosis in EGFR mutant patients whose median overall survival reached 28.1 months, significantly longer than the 14.9 months of EGFR wild-type patients. In the research by Porta et al. [15], PFS and OS of EGFR mutant group were 11.7 months and 12.9 months while of EGFR wild-type group 5.8 months and 3.1 months and the difference between the two groups was considered statistically significant. However some other researches on brain metastases hold different conclusions. In their retrospective study which enrolled 189 NSCLC patients, Hendriks et al. [8] found that the median time to brain metastase in EGFR mutant group and EGFR wild-type group was 20.8 months and 16.4 months respectively $(p=0.321)$; the median post brain metastases survival was 12.1 months and 10.7 months respectively $(\mathrm{p}=0.674)$, indicating that the incidence of brain metastases was not different between mutation group and wild-type group. Research by Krawczyk et al. [16] found the incidence of EGFR mutations in bone metastases was $75 \%$, significantly higher than in primary adenocarcinoma (12.8\%) and nervous system metastases (14.75\%). Overall survival of EGFR mutant patients with bone metastases was much longer than in patients without mutations $[7,8]$. Fujimoto et al. [7] revealed that the median overall survival was 22.7 months in EGFR mutant patients with bone metastases, significantly longer than the 7.7 months in EGFR wild-type group. Hendriks et al. [8] found that the median post metastases bone disease survival in EGFR mutant patients was 15.0 months, much longer than the 3.2 months in EGFR wild-type group $(\mathrm{p}=0.010)$.

In presented research 50 patients were assigned to EGFR mutation test and the median overall survival was 10.25 months in EGFR mutant patients, longer than the 8.75 months in EGFR wild-type patients. Among EGFR mutant patients, median overall survival was 7.50 months in solitary bone metastases, 10.50 months in solitary brain metastases and 11.50 months in combined bone and brain metastases, which was consistent with similar studies and indicated that radiotherapy could extend survival for patients with stage IV lung adenocarcinoma. However, different from previous studies, we detected no statistical difference in EGFR mutation status or metastatic sites. In contrast to this research, Fujimoto et al. [7], Hendriks et al. [8] and Porta et al. [15] administered active systemic treatment, without considering the effect of radiotherapy on survival. Indeed there are some researches 
indicative of a correlation between radiotherapy efficacy and EGFR mutation status. For instance, Johung et al. [9] found that brain metastases treated with gamma knife radiotherapy showed no in-field recurrence while patients without EGFR mutations exhibited higher local recurrence rate. Their multivariate analysis revealed that EGFR mutations status and lesion diameter were independently associated with local control after radiotherapy. In the research by Park et al. [17], $50 \%$ of EGFR mutant patients were assigned to radiation to the brain during EGFR-TKI treatment and the PFS of this group reached 12.6 months. In contrast to brain metastases, the overall survival of bone metastases was mainly associated with the number of metastatic lesions [18] and radiation doses $[13,14]$. According to Porta et al. [14] EGFR mutant patients could get benefit from EGFR-TKI and after radiotherapy subgroups treated with biological dose equivalent (BED) $\geq 40$ Gy had a favorable prognosis. In our research, brain metastases were administered radiation to the brain while bone metastases palliative radiotherapy, both combined with systemic approaches including EGFR-TKI and chemotherapy. The results showed improved life quality of patients, indicating active radiotherapy could affect disease development; both brain metastases and bone metastases with survival benefits, exhibiting no significant difference but further prospective study may be needed.

Bone metastases are commonly treated by combined modality therapy including chemotherapy, radiotherapy and EGFR-TKI. Some researches adopted EGFR-TKI for treatment of brain metastases despite of $\mathrm{BBB}$ and the plasma and cerebrospinal fluid (CSF) concentration of Gefitinib could reach $491.8 \pm 184.2 \mathrm{ng} / \mathrm{ml}$ and $6.2 \pm 4.6 \mathrm{ng} / \mathrm{ml}$ [10], and Erlotinib concentration in blood plasma and CSF could be $717.7 \pm 459.7$ and $23.7 \pm 13.4 \mathrm{ng} / \mathrm{ml}[11]$. One mice model test revealed that increased doses of Gefitinib brought higher concentration of the drug EGFR-TKI [19] which could have direct effect on metastatic cells in the brain. Research by Bokobza et al. [20] showed the combination of Gefitinib and radiotherapy didn't increase the killing of non-mutant cancer cells at the cellular level but still produced additive cell killing. Song et al. [3] indicated Gefitinib/Erlotinib could be used as salvage treatment to extend survival for patients with brain metastases who failed to respond to radiotherapy. In this research, EGFR mutant patients either received Erlotinib or Gefitinib, combined with radiotherapy, and the median overall survival were 10.25 months and 14.5 months respectively, presenting no significant difference. In their meta analysis, Liang et al. [21] found difference in efficacy between Gefitinib and Erlotinib, but they noted the overall survival could be influenced by other factors and EGFR-TKI T790M status in the population may have effects on the efficacy of TKI and cause bias. Radiotherapy worked in EGFR-TKI-resistant patients since it could directly damage cancer cell DNA [22], indicating combined modality treatment invovling TKI based chemotherapy and radiotherapy could enhance efficacy for Gefitinib-resistant patients. Probably because radiation had direct effect on cancer cell DNA, our research found no difference in overall survival between EGFR wild-type and EGFR mutant patients.

EGFR mutation test could affect clinical trial results. In a randomized trial carried out by Lee et al. [23], no statistically significant quality of life difference was found between patients treated by Erlotinib combined with whole brain radiation therapy (WBRT) and patients treated by WBRT only. Lee et al. believe it was because $45 / 80$ patients didn't receive EGFR mutation test and only $1 / 35$ was EGFR mutation positive. In our research, no statistical difference in metastatic sites was detected among the 65 patients untested for EGFR mutation status, similar to the result from EGFR mutant patients. However, the median overall survival in untested solitary bone metastases (8.50 months) was longer than in EGFR-mutant solitary bone metastases (7.50 months), which is different from comparisons between solitary brain metastases groups ( 8.50 vs 10.50 months) and between combined bone and brain metastases groups (9.50 vs 11.50 months). The reason for this statistical bias lies in that 65 patients didn't receive EGFR mutation test and 36 of them with unclear EGFR mutation status received EGFR-TKI treatment which could affect survival. It provides further evidence for the importance of EGFR mutation test for clinical research on lung adenocarcinoma.

In conclusion, presented research carried out a retrospective analysis and found that brain metastases and bone metastases had similar prognosis when patients with stage IV lung adenocarcinoma received early radiotherapy. EGFR mutant patients had longer survival than EGFR wild-type patients but the difference was not considered statistically significant, indicating that radiotherapy could extend survival time for wild-type patients. Radiotherapy combined with Gefitinib or Erlotinib have similar efficacy in treating stage IV lung adenocarcinoma. EGFR mutation test should be performed before treatment of stage IV lung adenocarcinoma, followed by a prospective clinical trial.

\section{References}

[1] RIIHIMAKI M1, HEMMINKI A2, FALLAH M3, THOMSEN H3, SUNDQUIST K4 et al.Metastatic sites and survival in lung cancer. Lung Cancer 2014; 86: 78-84. http://dx.doi. org/10.1016/j.lungcan.2014.07.020

[2] RODRIGUS P,DE BROUWER P, RAAYMAKERS E.Brain metastases and non-small cell lung cancer. Prognostic factors and correlation with survival after irradiation. Lung Cancer 2001; 32: 129-136. http://dx.doi.org/10.1016/S0169-5002(00)00227-0

[3] SONG, Z. and Y. ZHANG. Gefitinib and erlotinib for non-small cell lung cancer patients who fail to respond to radiotherapy for brain metastases. Journal of Clinical Neuroscience 2014; 21: s591-595. http://dx.doi.org/10.1016/j. jocn.2013.05.022

[4] SEQUIST LV, JOSHI VA, JANNE PA, MUZIKANSKY A, FIDIAS $P$ et al. Response to treatment and survival of patients with non-small cell lung cancer undergoing somatic EGFR 
mutation testing. Oncologist 2007; 12: p. 90-98. http://dx.doi. org/10.1634/theoncologist.12-1-90

[5] WON YW, HAN JY, LEE GK, PARK SY, LIM KY et al. Comparison of clinical outcome of patients with non-small-cell lung cancer harbouring epidermal growth factor receptor exon 19 or exon 21 mutations. Journal of Clinical Pathology, 2011. 64: 947-952. http://dx.doi.org/10.1136/jclinpath-2011200169

[6] MAK RH, DORAN E, MUZIKANSKY A, KANG J, NEAL JW . Outcomes after combined modality therapy for EGFR-mutant and wild-type locally advanced NSCLC. Oncologist 2011; 16: 886-895. http://dx.doi.org/10.1634/theoncologist.2011$\underline{0040}$

[7] FUIMOTO D, UEDA H, SHIMIZU R, KATO R, OTOSHI $\mathrm{T}$ et al. Features and prognostic impact of distant metastasis in patients with stage IV lung adenocarcinoma harboring EGFR mutations: importance of bone metastasis. Clinical \& Experimental Metastasis 2014; 31: 543-551. http://dx.doi. org/10.1007/s10585-014-9648-3

[8] HENDRIKS LE, SMIT EF, VOSSE BA, MELLEMA WW, HEIDEMAN DA, BOOTSMA GP et al. EGFR mutated nonsmall cell lung cancer patients: More prone to development of bone and brain metastases? Lung Cancer 2014; 84: 86-91. http://dx.doi.org/10.1016/j.lungcan.2014.01.006

[9] JOHUNG KL, YAO X, LI F, YU JB, GETTINGER SN et al. A clinical model for identifying radiosensitive tumor genotypes in non-small cell lung cancer. Clin Cancer Res 2013; 19: 55235532. http://dx.doi.org/10.1158/1078-0432.CCR-13-0836

[10] ZHAO J, CHEN M, ZHONG W, ZHANG L, LI L et al. Cerebrospinal Fluid Concentrations of Gefitinib in Patients With Lung Adenocarcinoma. Clinical Lung Cancer 2013; 14: 188-193. http://dx.doi.org/10.1016/j.cllc.2012.06.004

[11] DENG Y, FENG W, WU J, CHEN Z, TANG Y et al. The concentration of erlotinib in the cerebrospinal fluid of patients with brain metastasis from non-small-cell lung cancer. Molecular and Clinical Oncology 2014; 2: 116-120.

[12] ZENG L, CHOW E, BEDARD G, ZHANG L, FAIRCHILD A ET AL. Quality of Life After Palliative Radiation Therapy for Patients With Painful Bone Metastases: Results of an International Study Validating the EORTC QLQ-BM22. International Journal of Radiation Oncology Biology Physics 2012; 84: e337-e342. http://dx.doi.org/10.1016/j.ijrobp.2012.05.028

[13] CHOW E, VAN DER LINDEN YM, ROOS D, HARTSELL WF, HOSKIN $P$ et al.Single versus multiple fractions of repeat radiation for painful bone metastases: a randomised, controlled, non-inferiority trial.The Lancet Oncology 2014; 15: 164-171. http://dx.doi.org/10.1016/S1470-2045(13)70556-4

[14] KOMATSU T, KUNIEDA E, OIZUMI Y, TAMAI Y, AKIBA T.KOMATSU et al. An analysis of the survival rate after radiotherapy in lung cancer patients with bone metastasis: is there an optimal subgroup to be treated with high-dose radiation therapy? Neoplasma 2012; 59: 650-657. http://dx.doi. org/10.4149/neo 2012082

[15] PORTA R, SANCHEZ-TORRES JM, PAZ-ARES L, MASSUTI $\mathrm{B}$, REGUART $\mathrm{N}$ et al. Brain metastases from lung cancer responding to erlotinib: the importance of EGFR mutation. European Respiratory Journal 2011; 37: 624-631. http:// dx.doi.org/10.1183/09031936.00195609

[16] KRAWCZYK P, NICOS M, RAMLAU R, POWROZEK T, WOJAS-KRAWCZYK K ET AL. The Incidence of EGFR-Activating Mutations in Bone Metastases of Lung Adenocarcinoma.Pathology Oncology Research 2014; 20: 107-112. http://dx.doi.org/10.1007/s12253-013-9667-4

[17] PARK SJ, KIM HT, LEE DH, KIM KP, KIM SW et al.Efficacy of epidermal growth factor receptor tyrosine kinase inhibitors for brain metastasis in non-small cell lung cancer patients harboring either exon 19 or 21 mutation. Lung Cancer 2012; 77: 556-560. http://dx.doi.org/10.1016/j.lungcan.2012.05.092

[18] BAE HM, LEE SH, KIM TM, KIM DW, YANG SC et al.Prognostic factors for non-small cell lung cancer with bone metastasis at the time of diagnosis. Lung Cancer 2012; 77: 572-577. http://dx.doi.org/10.1016/j.lungcan.2012.05.094

[19] CHEN Y, WANG M, ZHONG W, ZHAO J.CHEN, Y et al. Pharmacokinetic and pharmacodynamic study of Gefitinib in a mouse model of non-small-cell lung carcinoma with brain metastasis.Lung Cancer 2013; 82: 313-318. http://dx.doi. org/10.1016/j.lungcan.2013.08.013

[20] BOKOBZA SM, JIANG Y, WEBER AM, DEVERY AM, RYAN AJ et al. Short-Course Treatment With Gefitinib Enhances Curative Potential of Radiation Therapy in a Mouse Model of Human Non-Small Cell Lung Cancer. International Journal of Radiation Oncology Biology Physics 2014; 88: 947-954. http://dx.doi.org/10.1016/j.ijrobp.2013.12.038

[21] LIANG W, WU X, FANG W, ZHAO Y, YANG Y et al. Network Meta-Analysis of Erlotinib, Gefitinib, Afatinib and Icotinib in Patients with Advanced Non-Small-Cell Lung Cancer Harboring EGFR Mutations. PLoS ONE 2014; 9: e85245. http:// dx.doi.org/10.1371/journal.pone.0085245

[22] DAS AK, SATO M, STORY MD, PEYTON M, GRAVES R et al. Non-Small Cell Lung Cancers with Kinase Domain Mutations in the Epidermal Growth Factor Receptor Are Sensitive to Ionizing Radiation. Cancer Research 2006; 66: 9601-9608. http://dx.doi.org/10.1158/0008-5472.CAN-06-2627

[23] LEE SM, LEWANSKI CR, COUNSELL N, OTTENSMEIER C, BATES A et al. Randomized Trial of Erlotinib Plus WholeBrain Radiotherapy for NSCLC Patients With Multiple Brain Metastases. JNCI Journal of the National Cancer Institute, 2014; 106: dju151. 